\title{
Myelodysplastic Syndrome, CTCAE
}

National Cancer Institute

\section{Source}

National Cancer Institute. Myelodysplastic Syndrome, CT CAE. NCI Thesaurus. Code C143690.

A disorder characterized by insufficiently healthy hematapoietic cell production by the bone marrow. 